\title{
A condensed-mass advection based model for the simulation of liquid polar stratospheric clouds
}

\author{
D. Lowe ${ }^{1}$, A. R. MacKenzie ${ }^{1}$, N. Nikiforakis ${ }^{2}$, and J. Kettleborough ${ }^{3}$ \\ ${ }^{1}$ Lancaster University, UK \\ ${ }^{2}$ DAMTP, Cambridge University, UK \\ ${ }^{3}$ Rutherford Appleton Laboratory, UK
}

Received: 22 March 2002 - Published in Atmos. Chem. Phys. Discuss.: 12 June 2002

Revised: 10 September 2002 - Accepted: 2 January 2003 - Published: 28 January 2003

\begin{abstract}
We present a condensed-mass advection based model (MADVEC) designed to simulate the condensation/evaporation of liquid polar stratospheric cloud (PSC) particles. A (Eulerian-in-radius) discretization scheme is used, making the model suitable for use in global or mesoscale chemistry and transport models (CTMs). The mass advection equations are solved using an adaption of the weighted average flux (WAF) scheme. We validate the numerical scheme using an analytical solution for multicomponent aerosols. The physics of the model are tested using a test case designed by Meilinger et al. (1995). The results from this test corroborate the composition gradients across the size distribution under rapid cooling conditions that were reported in earlier studies.
\end{abstract}

\section{Introduction}

PSCs have a major role in the destruction of stratospheric ozone, which they contribute to by activating chlorine and denitrifying the stratosphere. PSC particles enable the heterogeneous reactions which release active chlorine compounds from the man-made chlorofluorocarbons (CFCs) (Soloman et al., 1986). The rates of the heterogenous reactions are dependent on the physical state and composition of the particles (Carslaw and Peter, 1997). Recent analysis of space-borne observations (Tabazadeh et al., 2000) indicate that downward fluxes of large particles (greater than a few micrometres) can form within long-lived PSCs, denitrifying layers of the stratosphere and so increasing the life-time of active chlorine.

Correspondence to: D. Lowe (d.lowe@lancaster.ac.uk)
Field observations, both lidar and in situ, have shown that there are a number of different types of PSC particle, both solid and liquid, mainly composed of a mixture of $\mathrm{HNO}_{3} / \mathrm{H}_{2} \mathrm{SO}_{4} / \mathrm{H}_{2} \mathrm{O}$ (Peter, 1997). PSC particles form from the background stratospheric aerosol particles, which are mostly supercooled liquid $\mathrm{H}_{2} \mathrm{SO}_{4} / \mathrm{H}_{2} \mathrm{O}$ droplets, although many other minor components have been identified (Murphy et al., 1998). As the temperature falls below $200 \mathrm{~K}$, these droplets absorb $\mathrm{H}_{2} \mathrm{O}$ and $\mathrm{HNO}_{3}$, growing and changing in composition to become liquid supercooled ternary solution (STS) particles (Carslaw et al., 1994). Though the composition and mechanisms of formation and dynamics of these liquid particles are well known (Carslaw et al., 1997), the possible mechanisms of formation and different compositions of the solid PSC particles are still under study (e.g. Koop et al., 1997; Salcedo et al., 2000; Tolbert and Toon, 2001).

The aim of developing MADVEC (mass advection) model is to allow global or mesoscale CTMs to model the nonequilibrium evolution of PSC particles. PSC development has previously been studied using trajectory box models (i.e. Meilinger et al., 1995; Rizi and Visconti, 1999). However this type of model is unsuitable for use within CTMs, because the Lagrangian particle growth schemes used create gaps in the model "radius space". To avoid this problem we utilise a fixed size discretization, in which the aerosol population is described using mass distribution functions for each component (Pilinis, 1990).

We will initially describe the model, then discuss its performance and limitations. Analytical solutions to the aerosol general dynamic equation (GDE), developed by Fernández Díaz et al. (1999), will be used to validate the numerical solvers in the model. Then the PSC-specific physics of the model will be tested using a test case from Meilinger et al. (1995). 


\section{MADVEC model}

The particle distribution is described using a full-stationary size structure, which fixes the radius of each size bin, while particle growth is treated as advection of mass between size bins. Because of the fixed size bins, this method is ideal for nucleation, emissions, coagulation, and transport. However it suffers from numerical diffusion, and information about the original composition of growing particles is lost, which is a disadvantage when using particles with involatile components.

To avoid these problems models using full-moving size structures were developed (i.e. Gelbard, 1990; Meilinger et al., 1995). Particle growth causes the radii of the size bins to change, preserving particle information and eliminating numerical diffusion. However the lagrangian methods used can lead to gaps between size bins, causing problems for nucleation, coagulation and transport.

Recent work has focused on combining these two basic systems to reduce their disadvantages. Quasistationary size structures (Jacobson, 1997) use lagrangian particle growth, but then transpose the size bins back onto a stationary grid at each step, which can cause numerical diffusion. The movingcentre structure (Jacobson, 1997) uses fixed size bin edges, but with variable size bin centres, which change as the particles grow. Particle growth produces no diffusion, as all particles in the bin are moved at the same time, however some diffusion occurs when particles moved to a new bin are averaged with the particles already in the bin.

A full-stationary size structure is used in order that MADVEC can be as fast as possible while being compatiable with CTMs. A solver is chosen such that no spurious oscillations are generated in the neighborhood of steep gradients, and numerical diffusion is reduced. As yet the model calculates only the effects of condensation and evaporation on STS particles, no account is taken of nucleation, coagulation or sedimentation.

\section{$2.1 \quad$ Numerical basis}

The aerosol particle distribution is described with a set of partial differential equations derived from the aerosol GDE (Seinfeld and Pandis, 1998). For the case considered here, an isolated volume of dilute aerosol travelling with the air flow, the GDE can be written:

$\frac{\partial n(m, t)}{\partial t}+\frac{\partial}{\partial m}[I(m, t) n(m, t)]=S(m, t)$,

where $m$ is the mass of the particle, $n(m, t)$ is the size distribution density function at time $t$, such that $n(m, t) d m$ is the number concentration of particles in the mass interval $[m, m+d m] . I(m, t)$ is the rate of change of the total mass of a particle, $d m / d t$, due to condensation or evaporation. $S(m, t)$ is a source function describing particle formation and loss. The aerosol is considered dilute enough for no coagulation to occur.
For an aerosol without nucleation or primary sources, Eq. (1) translates to:

$\frac{\partial n(m, t)}{\partial t}+\frac{\partial}{\partial m}\left[n(m, t) \sum_{i=1}^{n c} \frac{d m_{i}}{d t}\right]=0$,

where $d m_{i} / d t$ is the rate of change of component $i$ in an individual particle, and $n c$ is the total number of components. This can be written in terms of a fractional growth rate, $H_{i}$ :

$\frac{d m_{i}}{d t}=H_{i} m$.

The mass concentration of component $i$ in the size range $[m, m+d m]$ can be defined as $q_{i}(m, t)$. The total aerosol distribution function, $q(m, t)$, is given by $q(m, t)=$ $\sum_{i=1}^{n} q_{i}(m, t)$, which is related to number concentration by:

$q(m, t)=m n(m, t)$.

Substituting Eqs. (3) and (4) into Eq. (2) gives us:

$\frac{\partial q(m, t)}{\partial t}+m \frac{\partial}{\partial m}[q(m, t) H(m, t)]=0$,

where $H=\sum_{i=1}^{n c} H_{i}$.

Equation (5) is a first order partial differential equation with characteristic curves $d m / d t=m H$. These characteristic curves are the curves following the mass growth of individual particles (or more precisely all the particles in the mass bin $m$ ). These provide a link with the Lagrangian methods of Meilinger et al. (1995). Pilinis (1990) has exploited the method of characteristics to derive the equations for each component from Eq. (5):

$\frac{\partial q_{i}}{\partial t}=H_{i} q-H q_{i}-m \frac{\partial q_{i} H}{\partial m}$.

Aerosol dynamic equations are more usually expressed with particle radius as a dependent variable. However, because particle distributions can cover large size ranges we adopt a logarithmic radius scale: $\mu=\ln r / r_{0}$, where $r_{0}$ is a reference radius. Then, the mass concentration of component $i, q_{i}(m, t)$, over mass range $[m, m+d m]$ is related to the mass concentration of component $i, p_{i}(\mu, t)$, over size range $[\mu, \mu+d \mu]$ by

$q_{i}(m, t) d m=p_{i}(\mu, t) d \mu$.

For a spherical particle

$\frac{d \mu}{d m}=\frac{1}{3 m}$.

Substituting Eqs. (7) and (8) into Eq. (6) gives us:

$\frac{\partial p_{i}}{\partial t}+\frac{1}{3} \frac{\partial}{\partial \mu}\left(p_{i} H\right)=H_{i} p ; \quad i=1, N$,

where $N$ is the number of species of which the particle is composed of (in the case below, three: $\mathrm{H}_{2} \mathrm{O}, \mathrm{HNO}_{3}$ and $\mathrm{H}_{2} \mathrm{SO}_{4}$ ). 
Pilinis (1990) solved these continuous distribution equations using the finite element method. However this scheme can produce negative aerosol concentrations close to the edge of square mode aerosol distribution. Dhaniyala and Wexler (1996) used a positive-definite scheme, which maintained positive concentrations while limiting numerical diffusion. We use a similar total variation diminishing (TVD) scheme, based on the weighted average flux (WAF) scheme.

\subsection{Numerical schemes}

Equation (9) can be solved by splitting it into two parts, allowing the use of the method of fractional steps (Yanenko, 1971; Toro, 1999, Chapter 15). Particle growth is described using a system of simultaneous ordinary differential equations

$$
\frac{d p_{i}}{d t}=H_{i} p ; \quad i=1, N
$$

While the consequent advection of mass in log-radius space ( $\mu$-space) is described using a system of partial differential equations

$$
\frac{\partial p_{i}}{\partial t}+\frac{1}{3} \frac{\partial}{\partial \mu}\left(p_{i} H\right)=0 ; \quad i=1, N
$$

The fractional step method advances the solution of Eq. (9) in one co-ordinante direction at a time. The advantage of this method is that it allows us to choose the most suitable schemes for solving each part of Eq. (9). However, it is limited by the need to use small time-steps, ensuring that the properties of the system do not appreciably change during the time-step.

The treatment of Eq. (11) as advection in $\mu$-space allows us to use fluid dynamical methods. The approach used is a TVD version of the basic WAF method (Toro, 1999, Chapter 13). This method calculates the flux of mass between adjacent size bins ( $\left.f_{i}^{\text {waf }}=p_{i} H\right)$, which is used to solve eq. (11) (see appendix for WAF equations). We solve Eq. (10) using a simple Euler forward step (an ode-timestep limiter is applied).

\subsection{Model physics}

Of the three components comprising STS particles, only $\mathrm{H}_{2} \mathrm{O}$ and $\mathrm{HNO}_{3}$ are volatile, $\mathrm{H}_{2} \mathrm{SO}_{4}$ is treated as involatile because of the low temperature of the stratosphere. Equilibrium vapour pressures of $\mathrm{H}_{2} \mathrm{O}$ and $\mathrm{HNO}_{3}$ above the STS particles are described using non-ideal solution thermodynamics following Luo et al. (1995). The bulk densities of the STS particles are calculated following MacKenzie et al. (1995).

The growth rates for each component can be determined by substituting for $d m_{i} / d t$ in Eq. (3). These are calculated assuming uncoupled mass fluxes between the gas and liquid phases for each component $i$ of the multicomponent particle (e.g. Vesala, 1991):

$$
\frac{d m_{i}}{d t}=a_{F S} \frac{4 \pi r M_{i} D}{R T}\left(e_{i}^{\infty}-a_{K}^{i} e_{i}^{v a p}\right),
$$

where $a_{F S}$ is the Fuchs-Sutugin correction, $M_{i}$ is the molar mass of component $i, D$ is the vapour diffusivity (assumed to be equal for each component) and $R$ is the universal gas constant. $e_{i}^{\infty}$ is the partial pressure of component $i$, initialised at the start of the model run and updated using the conservation equations: $e_{i}^{\infty}(t)+c \sum_{j=1}^{n b i n} p_{i}^{j}(t)=K$, where $K$ is a constant and $c$ is a conversion factor. The vapour pressure, $e_{i}^{v a p}$, of component $i$ is calculated from work by Luo et al. (1995). The Kelvin effect term for component $i$ is calculated from $a_{K}^{i}=\exp 2 \sigma v_{m}^{i} / R T r$, where $v_{m}^{i}$ is the partial molar volume of component $i$ in the liquid phase, and $T$ is the temperature. The liquid-gas surface tension, $\sigma$, is calculated from work by MacKenzie et al. (1995). Heat transport is not coupled with these mass fluxes.

\section{Analytical analysis}

Katoshevski and Seinfeld (1997) developed an analytical solution of the multicomponent aerosol general dynamic equation, which could be solved for condensationevaporation, deposition and sources. The condensationevaporation aspect of this solution was elaborated upon by Fernández Díaz et al. (1999), in order to study the complexities of this aspect of aerosol behaviour. They also presented several test cases. We shall use a variant of one of these below to validate our numerical model.

The analytical solution developed by Fernández Díaz et al. (1999) is also based on Pilinis's work (Eq. 9). They solved Eq. (9) for both $H=0$ and $H \neq 0$, and studied three growth laws: diffusion, surface reaction and volume reaction. We shall use their solutions for $H \neq 0$ and the diffusional growth law.

The growth rate for a particle of size $\mu$ is:

$\frac{d \mu}{d t}=\frac{1}{3} H$.

This equation defines a characteristic curve in the $(\mu, t)$ coordinate system. Equation (13) is integrated to obtain the equation of the characteristic curve:

$\mu=f\left(\mu_{0}, t_{0}, t\right)$

which may be inverted

$\mu_{0}=f_{0}\left(\mu, t_{0}, t\right)$,

where $\mu=\mu_{0}$ when $t=t_{0}$. This integration will be performed later for the diffusion case.

Using the method of characteristics, Fernández Díaz et al. solved Eq. (9) for $H \neq 0$ :

$$
\begin{gathered}
p_{i}(\mu, t)=\frac{H\left(f_{0}\left(\mu, t_{0}, t\right)\right)}{H(\mu)}\left(\left[\exp \left(3\left(\mu-f_{0}\left(\mu, t_{0}, t\right)\right)\right)-1\right]\right. \\
\left.\quad \times A_{i} p\left(f_{0}\left(\mu, t_{0}, t\right), t_{0}\right)+p_{i}\left(f_{0}\left(\mu, t_{0}, t\right), t_{0}\right)\right)
\end{gathered}
$$


where

$A_{i}=\frac{H_{i}(J, t)}{H(J, t)}$.

This analytical solution is valid while $A_{i}$ is constant, and $H_{i}$ is a function of separable variables $\left(H_{i}=H_{i}^{\mu}(\mu) H_{i}^{t}(t)\right)$.

Fernández Díaz et al. noted a limitation on the use of Eq. (16). When analyzing the evolution of an aerosol containing an evaporating component $\left(H_{i}<0\right)$ this equation will give negative values for $p_{i}$ after a certain time $\left(t_{\lim }^{i}\right)$, however until that time Eq. (16) is valid.

To simulate aerosol evolution a simple version of the diffusional growth law is used:

$H_{i}(s)=G_{i} h(s)$,

where $h(s)$ is a potential function and $G_{i}$ is a constant that depends on the condensing component. This ignores the Kelvin effect and fixes the concentration of each component in the vapour state (making $H_{i}$ independent of time). When all components possess the same growth law, we have

$H(s)=\left(\sum_{i=1}^{n c} G_{i}\right) h(s)=G h(s)$

and

$A_{i}=\frac{G_{i}}{\sum_{i=1}^{n c} G_{i}}=$ constant,

which allows us to use Eq. (16).

In diffusion-controlled growth the volume growth rate depends on the molar volume of the condensing species, on its vapour pressure, and on its molecular diffusion coefficient, and is nearly proportional to the particle diameter (see Sect. 2.3 above).

$\frac{d s_{i}}{d t}=G_{i} s^{1 / 3}$

hence

$H_{i}=G_{i} s^{-2 / 3}$.

If $G_{i}>0$ the component undergoes condensation, and if $G_{i}<0$ it undergoes evaporation.

If all the components condense or evaporate by diffusion then the growth law (Eq. 13) for the total particle becomes

$\frac{d \mu}{d t}=\frac{1}{3} G s_{r}^{-2 / 3} \exp (-2 \mu)$.

Equation (23) can then be integrated to give the equation of the characteristic curve (c.f. Eq. 15):

$\mu_{0}=f_{0}\left(\mu, t_{0}=0, t\right)=\frac{1}{2} \ln \left[\exp (2 \mu)-\frac{2}{3} G s_{r}^{-2 / 3} t\right]$.

\subsection{Test case}

As a test case we will use a three component aerosol with diffusion-controlled growth. Of the three components the first condenses, the second evaporates and the third is involatile:

$G_{1} s_{r}^{-2 / 3}=0.5, \quad G_{2} s_{r}^{-2 / 3}=-0.2, \quad G_{3} s_{r}^{-2 / 3}=0$.

giving a total growth rate:

$G s_{r}^{-2 / 3}=0.3$.

This case is similar to the actual PSC system we will be modelling, and so represents conditions which the model may have to deal with.

Our initial mass distribution consists of a single square mode:

$p^{0}(\mu)= \begin{cases}0, & \mu<-3, \\ 10, & -3 \leq \mu \leq 3, \\ 0, & 3<\mu .\end{cases}$

Between $\mu=-3$ and $\mu=3$, the masses of the individual components are given by:

$p_{3}^{0}=1$,

$p_{1}^{0}=\frac{p^{0}-p_{3}^{0}}{2}+\frac{\mu}{2}$,

$p_{2}^{0}=\frac{p^{0}-p_{3}^{0}}{2}-\frac{\mu}{2}$.

To ensure that $p_{2}$ remains positive the test run is analysed until $t=1.0\left(t_{\text {lim }}=2.09\right)$, allowing us to use Eq. (16). The test case is modelled in MADVEC using 100 size bins over the range $-6 \leq \mu \leq 6$.

Figures $1 \mathrm{a}$ and $\mathrm{b}$ illustrate the evolution of the total mass and the second component respectively.

The MADVEC model copes well with this square mode test, the analytical solution is reproduced almost exactly away from the edges of the mass distribution. Some numerical diffusion occurs at the discontinities, however these are minimal for both the VANLEER and SUPERBEE limiters (see appendix for details of the TVD limiters). Both solutions are positive throughout the size domain and integration time.

\section{Physical test case}

The physical components of MADVEC also require testing. To do this we will use the simple test case used by Meilinger et al. (1995) to demonstrate that significant concentration gradients can exist across the size distribution in a gravitywave-induced PSC.

Meilinger et al. (1995) used a trajectory box model to simulate the evolution of STS particles. Growth and evaporation were calculated using a Lagrangian scheme in radial 

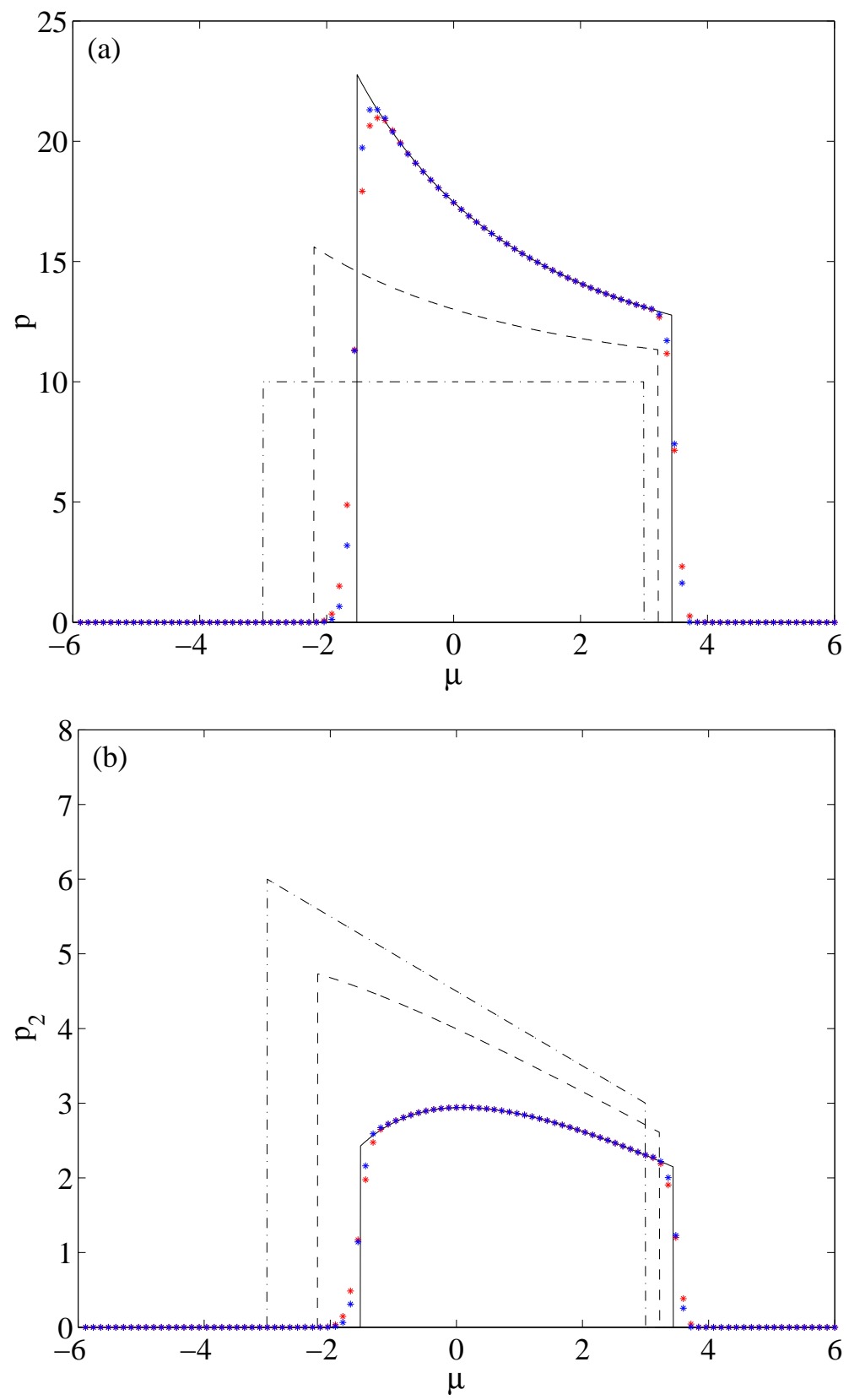

Fig. 1. Evolution of (a) the total mass distribution, and (b) the mass distribution of the second (evaporating) component, for the analytical test case. The black lines indicate the analytical solution at times: $(-\cdot-\cdot) t=0,(----) t=0.5$, and $(-) t=1.0$. The small asterisks indicate the distributions given by MADVEC at $t=1.0$, the colours indicate TVD limiter: red for VANLEER, and blue for SUPERBEE.

space, reducing numerical diffusion. The partial pressure and vapour pressures were calculated from Luo et al. (1995).

The test case simulates a mild lee wave cooling event (Fig. 2). The air mass initially cools adiabatically at a steady rate of $6 \mathrm{~K} \mathrm{hr}^{-1}$ from $196 \mathrm{~K}$ to $190 \mathrm{~K}$, remains at $190 \mathrm{~K}$ for an hour, then increases back to $196 \mathrm{~K}$ at the previous rate. Of the three components, $\mathrm{H}_{2} \mathrm{SO}_{4}$ is considered non-volatile, while the total mixing ratios of $\mathrm{H}_{2} \mathrm{O}$ and $\mathrm{HNO}_{3}$ are 5 ppmv and $10 \mathrm{ppbv}$ respectively, initial air pressure is $65 \mathrm{mbar}$. The starting particle distribution is lognormal, with total particle number density $N=10 \mathrm{~cm}^{-3}$, mode radius $\bar{r}=0.08 \mu \mathrm{m}$ and width $\sigma=1.8$. The physical test case is modelled in MADVEC using 50 size bins logarithmically distributed over the radius range $r=1.6 \mathrm{~nm}$ to $r=30 \mu \mathrm{m}$. Of these $50 \mathrm{bins}$, at any one time $30-40$ bins will contain particles. The WAF scheme is controlled using the vanleer TVD limiter. To enable comparision with MADVEC, Meilinger et al.'s data has been transposed onto the same fixed radius grid. 


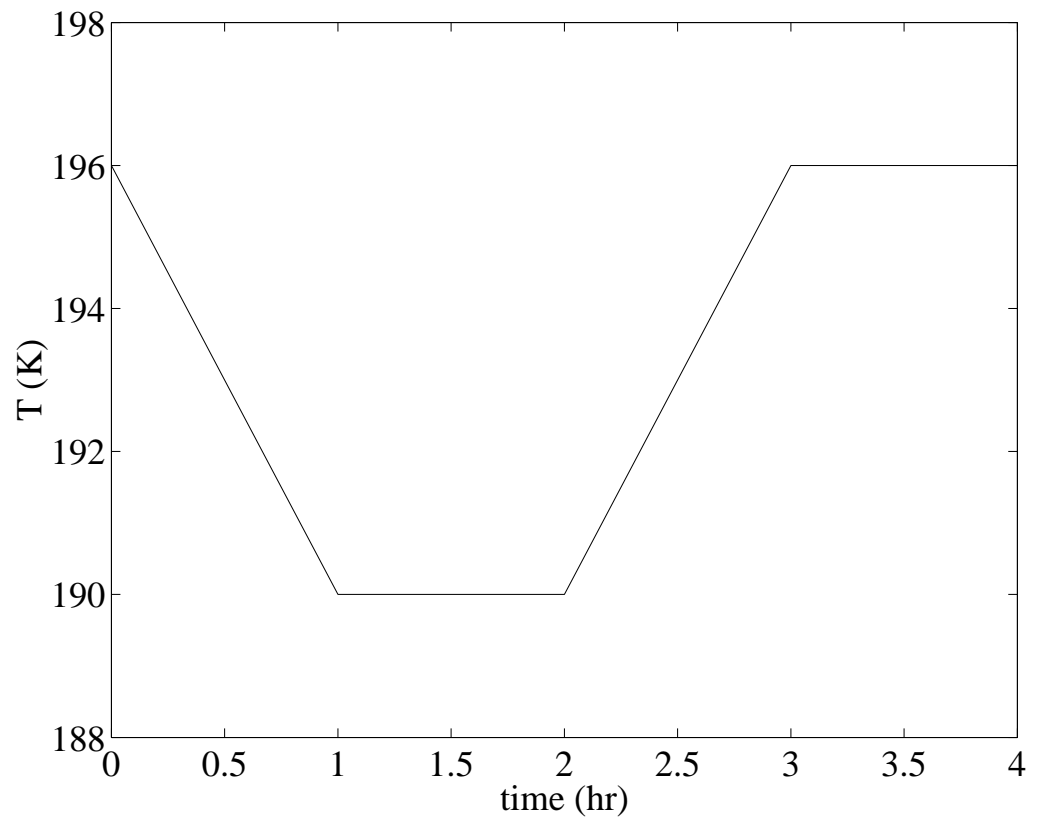

Fig. 2. Temperature profile of the adiabatic cooling event used by Meilinger et al. (1995).
Figures $3 \mathrm{a}$ and $\mathrm{b}$ show the development of $\mathrm{HNO}_{3}$ mass fractions during the test run for Meilinger et al. and MADVEC, respectively.

In both models particles smaller than $0.6 \mu \mathrm{m}$ have a composition close to a pure $\mathrm{HNO}_{3} / \mathrm{H}_{2} \mathrm{O}$ solution, however in MADVEC the $\mathrm{HNO}_{3}$ content of the larger particles $(\sim$ $2 \mu \mathrm{m}$ ) remains fairly constant, whereas in Meilinger et al. it increases to about $20 \%$.

The $\mathrm{HNO}_{3}$ mass fractions for particles of radius $0.16 \mu \mathrm{m}$ are shown in Fig. 4. As the temperature drops the $\mathrm{HNO}_{3}$ mass fractions of the models increase at the same rate. However at $190 \mathrm{~K}$, the $\mathrm{HNO}_{3}$ mass fractions determined by MADVEC are approximately 3\% lower than those reached by Meilinger et al.. As the temperature increases again so do the $\mathrm{HNO}_{3}$ mass fractions, as the particle composition follows the binary $\mathrm{HNO}_{3} / \mathrm{H}_{2} \mathrm{O}$ liquid curve. The maxima reached by MADVEC occurs earlier and is approximately $4 \%$ smaller than that of Meilinger et al..

The difference in $\mathrm{HNO}_{3}$ mass fractions between our results and those of Meilinger et al. (1995) is not sensitive to the resolution of MADVEC (tested by doubling the resolution of MADVEC relative to that used to produce Figs. $3 \mathrm{~b}$ and 4). Neither is there any indication of significant numerical diffusion when MADVEC is tested against the analytical solution (Figures 1a and b; but see also the discussion below). It is likely, therefore, that the difference between the models is due to small differences in physical parameters such as vapour pressure, density, surface tension, etc.

At high temperatures (above $193 \mathrm{~K}$ ), the size of STS particles is controlled by their $\mathrm{H}_{2} \mathrm{SO}_{4}$ content. Because $\mathrm{H}_{2} \mathrm{SO}_{4}$ is treated as inert the particle distribution should return to the original distribution upon returning to initial conditions.
However for mass-conserving schemes, like the one used in MADVEC, the mass of all components is mixed, which may lead to an unrealistic dispersion of mass in radius space. We combined three of the adiabatic cooling events described above into one single twelve hour run. The initial mass distribution, and the mass distributions after each hour spent at $196 \mathrm{~K}$ are plotted in Fig. 5.

Numerical dispersion occurs throughout the 12 hour run. While both ends of the distribution gain mass, the gain is more sustained in the smaller radius bins. The mass distribution also develops a skew towards the smaller radius bins, which, while there is no significant gain in mass, does result in an increase in particle number over the 12 hour run.

\section{Conclusions}

We have built a multi-component liquid aerosol box model with fixed size bins. The numerical methods used have been tested against analytical aerosol solutions, and been proven to work. The model physics have been tested using the test case published by Meilinger et al. (1995), and the results compared with those obtained by Meilinger et al. On the whole MADVEC reacts to changes in conditions in a similar manner to Meilinger et al.'s model, although the maximum $\mathrm{HNO}_{3}$ mass fractions reached by MADVEC are lower. At these maxima the aerosol particles practically have a binary $\mathrm{HNO}_{3} / \mathrm{H}_{2} \mathrm{O}$ composition. Heterogeneous freezing of $\mathrm{HNO}_{3} / \mathrm{H}_{2} \mathrm{O}$ particles close to the NAT stoichiometry $(\approx$ $54 \%$ ) may occur at temperatures above the ice point (Bogdan et al., submitted manuscript). This could be a possible mechanism of formation for the large $\mathrm{HNO}_{3}$-containing par- 

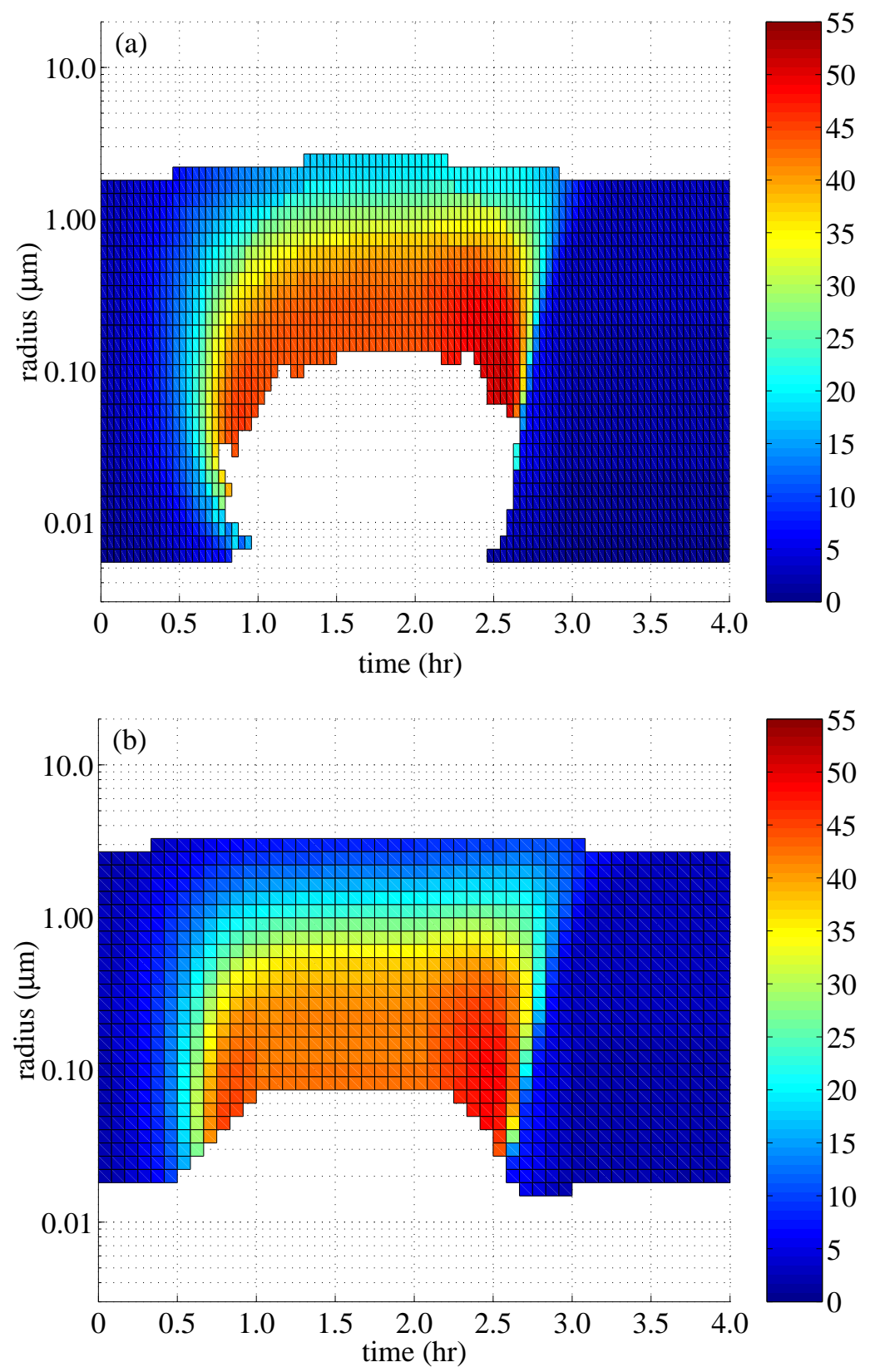

Fig. 3. Nitric acid mass fractions for (a) Meilinger et al.'s model, and (b) MADVEC, during the cooling event shown in Fig. 2. The scale bars on the left indicate the mass fraction $(\%)$. Note that the mass distribution produced by MADVEC has been cutoff at a minimum of $1 \mathrm{pgm}^{-3}$.

ticles which have been detected in the Arctic (Fahey et al., 2001).

Longer model runs have demostrated the stability of MADVEC, with little numerical diffusion occuring. Due to the fixed size distribution, MADVEC is suitable for use within global and mesoscale CTMs. However full nonequilibrium modelling of PSC particles will require high resolution parameterisation of mountain waves and of the PSCs created by them. Mesoscale models can, at high resolution, capture the larger scale features of mountain waves (Leut- becher and Volkert, 2000). However these models still fail to capture the smaller scale fluctuations (with wavelengths of a few $\mathrm{km}$ ), which have a large effect on the composition of STS particles (Voigt et al., 2000). Sedimentation of particles from PSCs, a proposed source of "NAT-rocks", also require higher vertical resolutions than those currently available (Fueglistaler et al., 2002). Further development of the parameterisation of these small-scale features is needed.

The four-hour test case used in this paper takes approximately $1000 \mathrm{CPU}$ seconds on a Sparc IIi $248 \mathrm{MHz}$ proces- 

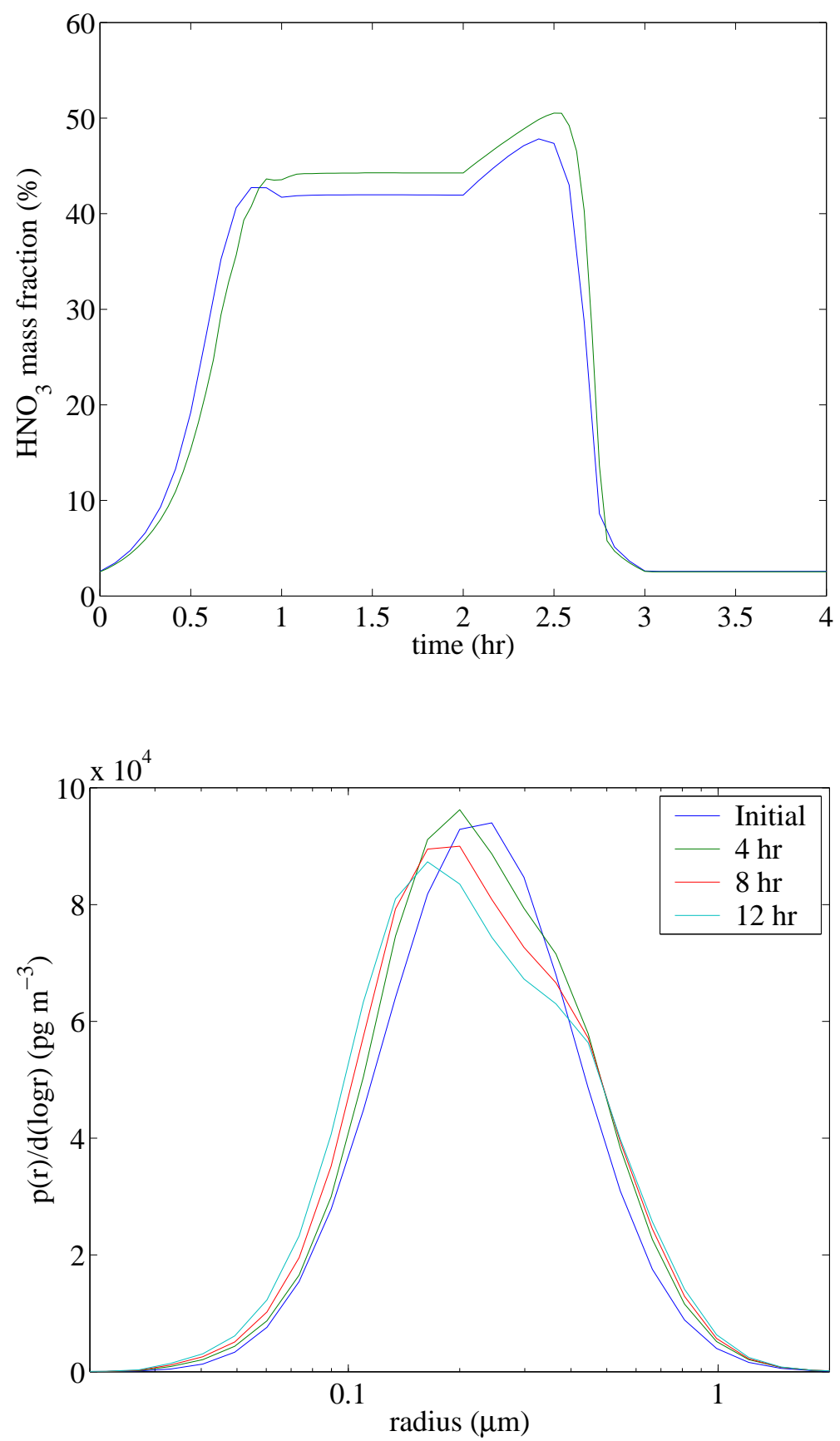

Fig. 4. Comparision of nitric acid mass fractions between Meilinger et al.'s model (green line) and MADVEC (blue line) for the radius bin of size $0.16 \mu \mathrm{m}$.

Fig. 5. Evolution of the total mass distribution over the period of $12 \mathrm{~h}$. Each distribution is taken after the model has spent an hour at a temperature of $196 \mathrm{~K}$.

sor. Reducing the resolution of the model (i.e. the number of size bins) can decrease the cost of the model by upto $30 \%$. It is envisaged that particles will be transported between grid cells using an adaption of the scheme used to advect trace gases. This would result in [number of components * number of size bin] tracers, thus at the resolution of 50 size bins the transport of 150 tracers would be required, while a 20-bin model would require 60 tracers. This will be expensive, but is achievable for the mesoscale modelling of mountain waves for a few days at a time.

\section{Appendix: The WAF Equations}

The WAF approach is a second-order implementation of the first-order Godunov method. The improvement on the order of accuracy is achieved by using a weighted average of the upwind and downwind contributions to the flux. The former controls the stability while the latter increases the order of accuracy.

Higher-than-first order schemes are not monotone and this will result in the appearance of spurious oscillations near 
high gradients. These can be eliminated by locally reducing the contribution of the downwind flux. This is achieved by introducing TVD functions into the WAF scheme.

The flux of mass between two size bins, $j$ and $j+1$, in $\mu$-space is given by

$f_{j+\frac{1}{2}}^{w a f}=\frac{1}{2}(1+c)\left(a_{j+\frac{1}{2}} p_{i}^{j}\right)+\frac{1}{2}(1-c)\left(a_{j+\frac{1}{2}} p_{i}^{j+1}\right)$,

where $a_{j+\frac{1}{2}}$ is the wave propagation speed, calculated as an average of the total growth rates for any two adjacent mass bins, and $p_{i}^{j}$ is the mass of component $i$ in size bin $j . c$ is the Courant-Friedrichs-Lewy (CFL) number, given by

$c=\frac{a_{j+\frac{1}{2}}}{\Delta \mu / \Delta t}$.

The CFL number can be thought of as the ratio of two speeds, the wave propagation speed and the grid speed, $\Delta \mu / \Delta t$, which is defined by the discretisation of the domain. This scheme is stable providing that the following condition is fulfilled

$0 \leq|c| \leq 1$.

Flux limiting is achieved by introducing a new parameter $\phi$, and Eq. (25) is rewritten as

$f_{j+\frac{1}{2}}=\frac{1}{2}(1+\phi)\left(a_{j+\frac{1}{2}} p_{i}^{j}\right)+\frac{1}{2}(1-\phi)\left(a_{j+\frac{1}{2}} p_{i}^{j+1}\right)$,

where

$\phi_{j+\frac{1}{2}}=\phi_{j+\frac{1}{2}}\left(r_{j+\frac{1}{2}},|c|\right)$

and $r_{j+\frac{1}{2}}$ is a measure of the gradient of the advected quantity. It is used to adjust $\phi$ depending on local conditions in the data, and is defined as the ratio of the upwind change to the local change

$r_{j+\frac{1}{2}}=\frac{\Delta_{u p w}}{\Delta_{l o c}}$,

where

$r_{j+\frac{1}{2}}= \begin{cases}\frac{p_{i}^{j}-p_{i}^{j-1}}{p_{i}^{j+1}-p_{i}^{j}} & \text { if } a_{j+\frac{1}{2}}>0, \\ \frac{p_{i}^{j+2}-p_{i}^{j+1}}{p_{i}^{j+1}-p_{i}^{j}} & \text { if } a_{j+\frac{1}{2}}<0 .\end{cases}$

The WAF limiter function, $\phi_{j+\frac{1}{2}}\left(r_{j+\frac{1}{2}},|c|\right)$, is related to any given conventional flux limiter, $\psi_{j+\frac{1}{2}}(r)$ by

$\phi_{j+\frac{1}{2}}\left(r_{j+\frac{1}{2}},|c|\right)=1-(1-|c|) \psi_{j+\frac{1}{2}}(r)$.

In this work we will use two standard flux limiter functions, given below with subscripts removed for brevity: SUPERBEE is given by

$\psi_{s b}(r)= \begin{cases}0 & \text { if } r \leq 0, \\ 2 r & \text { if } 0 \leq r \leq \frac{1}{2}, \\ 1 & \text { if } \frac{1}{2} \leq r \leq 1, \\ r & \text { if } 1 \leq r \leq 2, \\ 2 & \text { if } r \geq 2,\end{cases}$ and VANLEER is given by

$\psi_{v l}(r)= \begin{cases}0 & \text { if } r \leq 0, \\ \frac{2 r}{1+r} & \text { if } r \geq 0 .\end{cases}$

Acknowledgements. Original developments on MADVEC were made in Centre for Atmospheric Science at Cambridge University, funded by the Isaac Newton Trust and EC contract ENV4 CT97 0533. DL is funded by NERC, award number GT/04/99/AS/107. Our thanks to Stefanie Meilinger for providing us with a copy of her model data.

\section{References}

Bogdan, A., Kulmala, M., MacKenzie, A. R., Laaksonen, A., Molina, M. J., and Avramenko, N.: The study of finely divided aqueous systems as an aid to understanding the formation mechanism of PSCs: 1 . the case of $\mathrm{HNO}_{3} / \mathrm{H}_{2} \mathrm{O}$ and $\mathrm{H}_{2} \mathrm{SO}_{4} / \mathrm{H}_{2} \mathrm{O}$ systems, J. Geophys. Res., submitted, 2002.

Carslaw, K. S., Peter, T., and Clegg, S. L.: Modeling the composition of liquid stratospheric aerosols, Rev. Geophys., 35, 125-154, 1997.

Carslaw, K. S. and Peter, T.: Uncertainties in reactive uptake coefficients for solid stratospheric particles - 1. surface chemistry, Geophys. Res. Lett., 24, 1743-1746, 1997.

Carslaw, K. S., Luo, B. P., Clegg, S. L., Peter, T., Brimblecombe, P., and Crutzen, P. J.: Stratospheric aerosol growth and $\mathrm{HNO}_{3}$ gas phase depletion from coupled $\mathrm{HNO}_{3}$ and water uptake by liquid particles, Geophys. Res. Lett., 21, 2479-2482, 1994.

Dhaniyala, S. and Wexler, A. S.: Numerical schemes to model condensation and evaporation of aerosols, Atmos. Environ., 30, 919928, 1996.

Fahey, D. W., Gao, R. S., Carslaw, K. S., Kettleborough, J., Popp, P. J., Northway, M. J., Holecek, J. C., Ciciora, S. C., McLaughlin, R. J., Thompson, T. L., Winkler, R. H., Baumgardner, D. G., Gandrud, B., Wennberg, P. O., Dhaniyala, S., McKinney, K., Peter, T., Salawitch, R. J., Bui, T. P., Elkins, J. W., Webster, C. R., Atlas, E. L., Jost, H., Wilson, J. C., Herman, R. L., Kleinböhl, A., and von König, M.: The detection of large $\mathrm{HNO}_{3}$-containing particles in the winter Artic stratosphere, Science, 291, 1026-1031, 2001.

Fernández Díaz, J. M., Rodríguez Braña, M. A., Arganza García, B., González-Pola Muñiz, C., and García Nieto, P. J.: Difficulties inherent to the use of analytic solution of the condensationevaporation equation for multicomponent aerosols, Atmos. Environ., 33, 1245-1259, 1999.

Fueglistaler, S., Luo, B. P., Voigt, C., Carslaw, K. S., and Peter, Th: NAT-rock formation by mother clouds: a microphysical model study, Atmos. Chem. Phys., 2, 93-98, 2002.

Gelbard, F.: Modeling multicomponent aerosol particle growth by vapor condensation, Aero. Sci. Tech., 12, 399-412, 1990.

Jacobson, M.Z.: Development and application of a new air pollution modeling system - ii. aerosol module structure and design, Atmos. Environ., 31, 131-144, 1997.

Katoshevski, D. and Seinfeld, J. H.: Analytical solution of the multicomponent aerosol general dynamic equation - without coagulation, Aero. Sci. Tech., 27, 541-549, 1997.

Koop, T., Luo, B. P., Biermann, U. M., Crutzen, P. J., and Peter, T.: Freezing of $\mathrm{HNO}_{3} / \mathrm{H}_{2} \mathrm{SO}_{4} / \mathrm{H}_{2} \mathrm{O}$ solutions at stratospheric tem- 
peratures: Nucleation statistics and experimants, J. Phys. Chem. A, 101, 1117-1133, 1997.

Leutbecher, M., and Volkert, H.: The propagation of mountain waves into the stratosphere: quantitative evaluation of threedimensional simulations, J. Atmos. Sci., 57, 3090-3108, 2000.

Luo, B., Carslaw, K. S., Peter, T., and Clegg, S. L.: Vapour pressures of $\mathrm{H}_{2} \mathrm{SO}_{4} / \mathrm{HNO}_{3} / \mathrm{HCl} / \mathrm{HBr} / \mathrm{H}_{2} \mathrm{O}$ solutions to low stratospheric temperatures, Geophys. Res. Lett., 22, 247-250, 1995.

MacKenzie, A. R., Kulmala, M., Laaksonen, A., and Vesala, T.: On the theories of type 1 polar stratospheric cloud formation, J. Geophys. Res., 100, $11275-11288,1995$.

Meilinger, S. K., Koop, T., Luo, B.P., Huthwelker, T., Carslaw, K. S., Krieger, U., Crutzen, P. J., and Peter, T.: Size-dependent stratospheric droplet composition in lee wave temperature fluctuations and their potential role in PSC freezing, Geophys. Res. Lett., 22, 3031-3034, 1995.

Murphy, D. M., Thomson, D. S., and Mahoney, M. J.: In situ measurements of organics, meteoritic material, mercury, and other elements in aerosols at 5 to 19 kilometers, Science, 282, 16641669, 1998.

Peter, T.: Microphysics and heterogeneous chemistry of polar stratospheric clouds, Annu. Rev. Phys. Chem., 48, 785-822, 1997.

Pilinis, C.: Derivation and numerical solution of the species mass distribution equations for multicomponent particulate systems, Atmos. Environ., 24A, 1923-1928, 1990.

Rizi, V. and Visconti, G.: Physical analogs and performance of a box model for composition and growth of $\mathrm{H}_{2} \mathrm{SO}_{4} / \mathrm{H}_{2} \mathrm{O}$ and $\mathrm{HNO}_{3} / \mathrm{H}_{2} \mathrm{SO}_{4} / \mathrm{H}_{2} \mathrm{O}$ aerosol on the stratosphere, J. Aero. Sci., 30, 1095-1113, 1999.

Salcedo, D., Molina, L.T., and Molina, M. J.: Nucleation rates of nitric acid dihydrate in $1: 2 \mathrm{HNO}_{3} / \mathrm{H}_{2} \mathrm{O}$ solutions at stratospheric temperatures, Geophys. Res. Lett., 27, 193-196, 2000.

Seinfeld, J.H. and Pandis, S. N.: Atmospheric Chemistry and Physics, Wiley, New York, 1998.

Soloman, S., Garcia, R. R., Rowland, F. S., and Wuebbles, D. J.: On the depletion of Antartic ozone, Nature, 321, 755-758, 1986.

Tabazadeh, A., Santee, M.L., Danilin, M. Y., Pumphrey, H.C., Newman, P. A., Hamill, P. J., and Mergenthaler, J.L.: Quantifying denitrification and its effect on ozone recovery, Science, 288, 1407-1411, 2000.

Tolbert, M. A. and Toon, O. B.: Solving the PSC mystery, Science, 292, 61-63, 2001.

Toro, E. F.: Riemann Solvers and Numerical Methods for Fluid Dynamics, Springer, Berlin, 1999.

Vesala, T.: Binary droplet evaporation and condensation as phenomenological processes, Commentationes PhysicoMathematicae et Chemico-Medicae, 127, 1991.

Voigt, C., Tsias, A., Dörnbrack, A., Meilinger, S., Luo, B., Schreiner, J., Larsen, N., Mauersbergerk K., and Peter, T.: Nonequilibrium compositions of liquid polar stratospheric clouds in gravity waves, Geophys. Res. Lett., 27, 3873-3876, 2000.

Yanenko, N. N.: The Method of Fractional Steps, Springer, Berlin, 1971. 